\title{
A Paradoxical Chemoresistance and Tumor Suppressive Role of Antioxidant in Solid Cancer Cells: A Strange Case of Dr. Jekyll and Mr. Hyde
}

\author{
Jolie Kiemlian Kwee \\ Coordenação de Pesquisa, Instituto Nacional de Câncer, Rua André Cavalcante 37, 20231-050, Rio de Janeiro, RJ, Brazil \\ Correspondence should be addressed to Jolie Kiemlian Kwee; jkwee@inca.gov.br
}

Received 5 December 2013; Revised 16 March 2014; Accepted 17 March 2014; Published 3 April 2014

Academic Editor: David Vauzour

Copyright (c) 2014 Jolie Kiemlian Kwee. This is an open access article distributed under the Creative Commons Attribution License, which permits unrestricted use, distribution, and reproduction in any medium, provided the original work is properly cited.

\begin{abstract}
Modulation of intracellular antioxidant concentration is a double-edged sword, with both sides exploited for potential therapeutic benefits. While antioxidants may hamper the efficacy of chemotherapy by scavenging reactive oxygen species and free radicals, it is also possible that antioxidants alleviate unwanted chemotherapy-induced toxicity, thus allowing for increased chemotherapy doses. Under normoxic environment, antioxidants neutralize toxic oxidants, such as reactive oxygen species (ROS), maintaining them within narrow boundaries level. This redox balance is achieved by various scavenging systems such as enzymatic system (e.g., superoxide dismutases, catalase, and peroxiredoxins), nonenzymatic systems (e.g., glutathione, cysteine, and thioredoxin), and metal-binding proteins (e.g., ferritin, metallothionein, and ceruloplasmin) that sequester prooxidant metals inhibiting their participation in redox reactions. On the other hand, therapeutic strategies that promote oxidative stress and eventually tumor cells apoptosis have been explored based on availability of chemotherapy agents that inhibit ROS-scavenging systems. These contradictory assertions suggest that antioxidant supplementation during chemotherapy treatment can have varied outcomes depending on the tumor cellular context. Therefore, understanding the antioxidant-driven molecular pathways might be crucial to design new therapeutic strategies to fight cancer progression.
\end{abstract}

\section{Introduction}

Reactive oxygen and nitrogen species (ROS/RNS) are oxidants natural products formed during cell vital metabolism activity that orchestrate the transmission of regulatory signals for proliferation, migration, defence, vasorelaxation, autophagy, and apoptosis signals (Figure 1(a)) [1-12]. Progress in redox biochemistry study has revealed an oxygen adaptation, whereby the cell has acquired the capability to initiate changes to the local redox environment as a means of regulating signaling pathways $[1-4,6-11]$. This has changed the way cellular oxidant production is viewed, from a simplistic model where all oxidant production is inherently damaging to a more complex scenario where a regulated small increase in oxidant production can be essential for optimal cellular function (Figure 1) [1-12]. In this model ROS and RNS act as second messengers, forming an integral part of the signal transduction network [1-4, $9,11,12]$. Reactive nitrogen species are produced by the endothelium inducing vascular relaxation when vascular smooth muscle cells were stimulated with vasodilators such as acetylcholine, histamine, and bradykinin. Nitric oxide synthase catalyzes a five-electron oxidation of a guanidine nitrogen of L-arginine in the formation of citrulline and nitric oxide $[7-9,11,12]$. On the other hand, ROS are heterogeneous group diatomic oxygen derived of free and nonfree radicals species with a wide range of reactivity [1012]. Their formation begins with the univalent reduction of oxygen to produce superoxide radical $\left(\mathrm{O}_{2}{ }^{--}\right)$, a free radical that gives rise to many highly reactive species such as hydroperoxyl radical $\left(\mathrm{HO}_{2}{ }^{\circ}\right)$, hydrogen peroxide $\left(\mathrm{H}_{2} \mathrm{O}_{2}\right)$, and hydroxyl radical $\left({ }^{\circ} \mathrm{OH}\right)$ (Figure 2$)$ [10-12]. For example, superoxide can dismutate to form hydrogen peroxide $\left(\mathrm{H}_{2} \mathrm{O}_{2}\right)$, a membrane-permeable, mildly prooxidant molecule which in turn can lead to formation of several highly oxidizing derivatives such as hydroxyl radicals (Figure 2). Also, $\mathrm{O}_{2}{ }^{--}$ can react with nitric oxide $\left(\mathrm{NO}^{\circ}\right)$ resulting in peroxynitrite $\left(\mathrm{OONOO}^{-}\right)$, a high RNS (Figure 2) [11, 12]. Mitochondria 

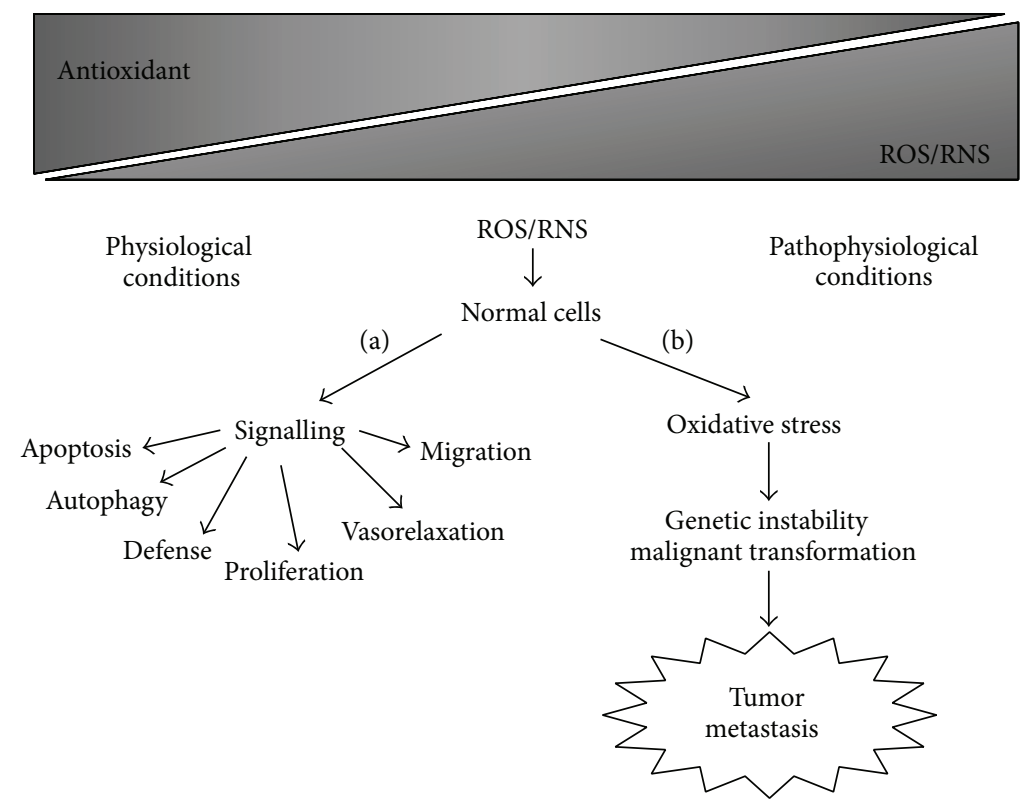

FIGURE 1: Schematic representation of reactive oxygen and nitrogen species (ROS/RNS) inductions in physiological (a) and pathophysiological (b) conditions.

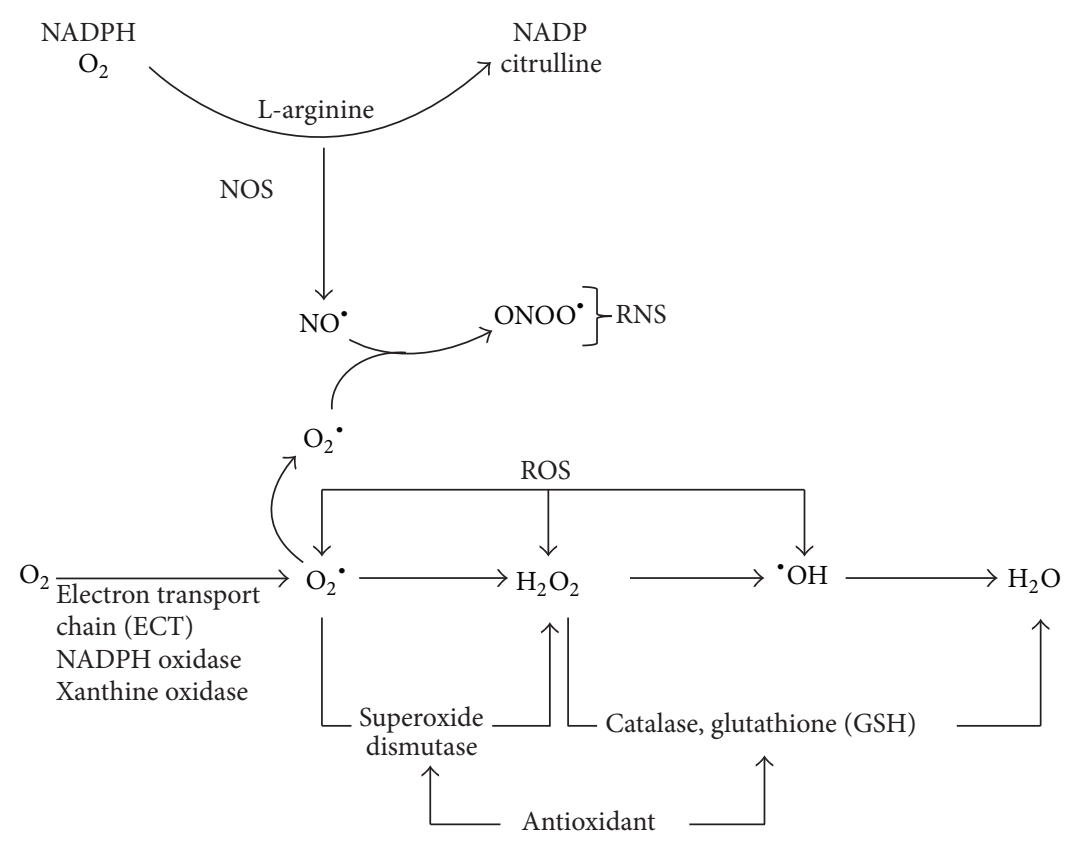

FIGURE 2: Sources of reactive oxygen (ROS) and nitrogen (RNS) species production. Enzymatic and nonenzymatic antioxidants counterbalance it.

form the major powerhouse of ROS production; they are generated in association with the activity of the respiratory chain such as NADH dehydrogenase enzyme complexes in aerobic ATP production [13-15]. In addition, two classic phagocytic ROS-generating enzymes use molecular oxygen as a substrate, including the multisubunit NADPH oxidase and its homologue NOX/Duox family and myeloperoxidase in various tissues in response to extracellular influences $[16,17]$. Other sources of ROS production include the cytochrome P450 (CYP450) system, which is involved mainly in removing or detoxifying toxic substances in the liver [13] and xanthine oxidase which catalyzes the oxidation of hypoxanthine to xanthine with the formation of $\mathrm{H}_{2} \mathrm{O}_{2}$ [18]. The imbalance of this cellular redox state is characteristic of many diseases where abnormal oxidant production causes extensive tissue damage (Figure 1(b)) $[3,6,19]$. Antioxidant has been defined as any substance that significantly delays or prevents oxidative damage of an oxidizable substrate (Figure 2). Due to their high reactivity, the ROS production levels are tightly controlled by antioxidants to avoid oxidative 

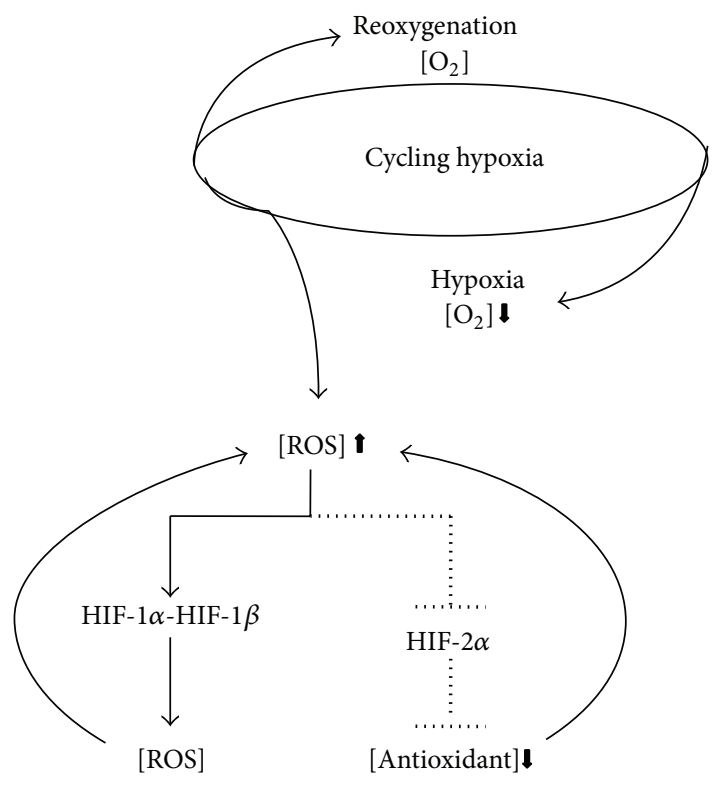

FIGURE 3: Schematic representation of cycling hypoxia effects on ROS production through activities modulations of HIF- $1 \alpha$ and HIF$2 \alpha$.

stress and, eventually, oxidative damage which is frequently linked to genetic instability, tumor promotion, and metastasis (Figure 1) [20]. On the other hand, the primary mechanism of many chemotherapy drugs and ionizing radiation, widely used against cancer cells, is the formation of ROS $[11,21]$. At this point, questions arise whether reduction of oxidative stress in tumor cell environment with antioxidant treatment would be beneficial or not [22]. Moreover, it should be stressed that the antioxidants cannot distinguish between the radicals that play a beneficial role and those that cause carcinogenesis. Understanding the biological redox system for the development of more effective and less toxic chemotherapy ROS induction strategies for cancer cells is deserved [21, 22]. Therefore, the modulation of intracellular antioxidant concentration is a double-edged sword, with both sides exploited for potential therapeutic benefits.

\section{ROS and Hypoxia in Solid Tumors}

Solid tumors are known to have a poor microvascular network and high interstitial fluid pressure resulting in hypoxic environment conferring chemo and radiotherapy resistance [22]. There are three major forms of hypoxia that varies with the duration: acute, chronic, and intermittent. Acute hypoxia occurs when tumor vessels become temporarily hypoxic for a period of seconds or a few hours. Chronic hypoxia is a progressive and severe reduction in oxygen (hours to days) [22]. Intermittent hypoxia, also referred to as cycling hypoxia, is characterized by cyclic periods of hypoxia and reoxygenation and plays the main role in resistance of solid tumor treatments (Figure 3) [23-26]. Hypoxic microenvironments are characterized by extreme heterogeneities in tumor cells oxygenation that arise as a result of the increased oxygen diffusion distance due to tumor expansion and poorly devel-

oped vascular networks [22, 27]. Gradients in oxygen are frequently found surrounding perfused vessels, ranging from normal values near the blood vessel to complete anoxia adjacent to necrosis $[27,28]$. The balanced proportion of hypoxic cells in cancer is driven by the tolerance of individual cells to these different types of hypoxia and varies remarkably among different tumors with otherwise similar clinical features [29]. These differences are important, because the fraction of viable hypoxic cells is a major determinant of prognosis, as hypoxic cells are highly resistant to chemotherapy and radiation therapy (Figure 3). Reducing cellular tolerance to hypoxia is therefore a strategy to reduce the proportion of hypoxic cells in tumors to improve current cancer therapy [27-33]. Tumor cells can adapt to hypoxic conditions by employing a variety of survival tools, which result in the promotion of cancer cell growth and metastasis [22, 32]. This adaptation is mainly mediated by hypoxia-inducible factor-1 (HIF-1) (Figure 3). HIF-1 is a heterodimeric transcription factor consisting of an oxygen-regulated subunit (HIF- $1 \alpha$ ) and a stable nuclear factor, HIF-1 $\beta$ aryl hydrocarbon receptor nuclear translocator (ARNT). Under normoxic conditions, HIF- $1 \alpha$ is hydroxylated by prolyl hydroxylase (PHD) at proline 402 and proline 564, and the hydroxylated HIF-1 $\alpha$ recruits von Hippel-Lindau (pVHL), an E3 ubiquitin protein ligase, and is rapidly degraded by the proteasome after being targeted for ubiquitination (Figure 3). Under hypoxic conditions, cytosolic HIF-1 $\alpha$ is stabilized by inhibition of the oxygen- and PHD-dependent enzymatic hydroxylation of proline residues and subsequently translocated to the nucleus, where it binds HIF- $1 \beta[30,34,35]$. The complex binds to the hypoxiaresponse element in its targets, which results in the transactivation of numerous genes encoding proteins necessary for the blood supply, energy production, growth/survival, invasion/metastasis, and chemo/radioresistance (Figure 4) $[30,35]$. An association of HIF-1 $\alpha$ overexpression with cell proliferation and poor prognosis has been observed in many kinds of human cancers $[30,34,35]$. It is well known that hypoxic conditions increase intracellular ROS levels [14] and recent studies provide important insights into the molecular mechanisms by which cycling hypoxia increases the oxidative stress [24]. This constant generation of ROS through intensive cycling hypoxia stabilizes HIF-1 $\alpha$ by preventing its degradation and induces HIF- $2 \alpha$ degradation (Figure 3 ). Since HIF$1 \alpha$ regulates genes encoding prooxidant enzymes and HIF$2 \alpha$ is a potent regulator of the genes encoding antioxidant enzymes, it was proposed that both HIFs contribute in part to the oxidative stress caused by cycling hypoxia. [36-39]. Ironically the main mechanism of ionizing irradiation and many anticancer drugs to induce apoptosis is through ROS which activate HIF- $1 \alpha[11,30]$.

\section{ROS and Chemotherapeutic Drugs}

Despite great improvements in screening strategies and adjuvant therapies, current treatments still rely heavily on conventional chemotherapy for most cancers. Additionally, most of these conventional chemotherapies agents such as taxanes, anthracyclines, and platinum coordination complexes induce ROS [11, 40-42] and are somehow cardiotoxic [43, 44]. 


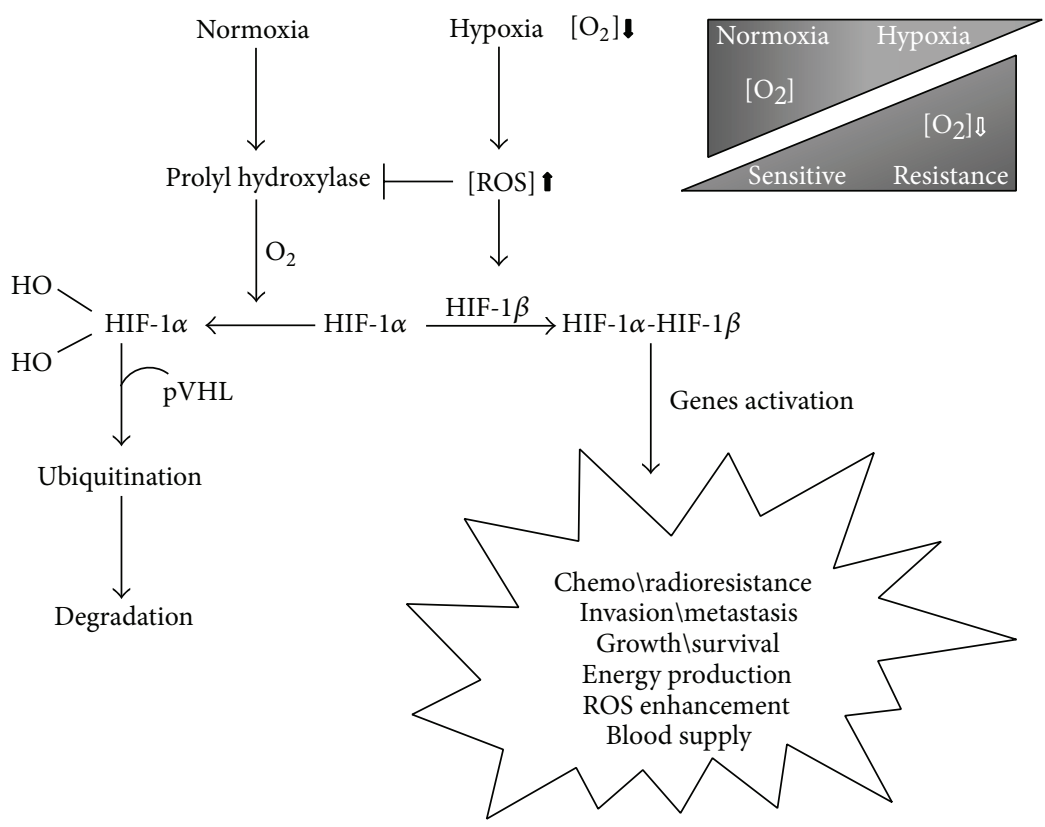

FIGURE 4: Role of ROS in hypoxia and normoxia.

Hence, the efficacy of these prooxidant chemotherapeutic agents is dose-dependent, which is limited by toxicity to nontumor tissues, as a result of its poor tumor selectivity. Modulation of ROS levels by antioxidants may be effective in protecting nontumor tissues especially the heart from oxidative damage but they may also reduce the efficacy of these anticancer drugs [43]. Nevertheless, the mechanism by which these chemotherapeutic agents inducers exhibit antitumor effects is likely multifactorial. Consequently, to improve survival length and preserve quality of life, the challenge is to develop approaches aimed at increasing chemotherapy toxicity to tumor tissue while not affecting nontumor tissues $[43,44]$. Therefore, the degree to which ROS contribute to the antineoplastic effects of these chemotherapeutic drugs should be evaluated.

\section{Antioxidants Playing Hyde and Jekyll}

4.1. Exogenous Antioxidant. In order to maintain an appropriate level of ROS and regulate their action, the body's natural defense against oxidative stress consists of several antioxidative systems. Therefore, mammalian cells have developed many enzymatic and nonenzymatic antioxidative systems $[20,45,46]$ as well as transfer proteins that sequester prooxidant metals inhibiting their participation in redox reactions (Table 1) [47]. Components of the endogenous antioxidant defense system work together and in concert with dietary antioxidants (Table 2) $[20,21,46]$ to prevent and reduce oxidative stress. In addition, the antioxidant activity of many of these enzymes and compounds is reliant upon minerals derived from the diet such as selenium, copper, manganese, and zinc (Table 2) [48]. Much debate has focused on the use of antioxidant supplements by patients undergoing chemotherapy due to concerns that the antioxidants may interfere with the mechanism of action of the therapeutic
TABLE 1: Endogenous antioxidants.

\begin{tabular}{ll}
\hline Endogenous antioxidants & Examples \\
\hline \multirow{2}{*}{ Enzymes } & Superoxide dismutase \\
& Catalase \\
& Peroxiredoxins \\
& Glutathione peroxidase \\
GSH enzyme-linked system & Glutathione S-transferase \\
& Glutathione reductase \\
& Glutathione \\
Nonenzymes & Cysteine \\
& Thioredoxin \\
& Ferritin \\
Metal-binding proteins & Metallothionein \\
& Ceruloplasmin \\
\hline
\end{tabular}

TABLE 2: Exogenous antioxidants.

\begin{tabular}{llc}
\hline & \multicolumn{2}{c}{ Example of exogenous antioxidants } \\
\hline Vitamin $\mathrm{C}$ & $\longrightarrow$ & Ascorbate/ascorbic acid \\
Vitamin E & $\longrightarrow$ & Tocopherols, tocotrienols \\
Carotenoids & $\longrightarrow$ & $\alpha$-carotene, $\beta$-carotene, lycopene \\
Polyphenols & $\longrightarrow$ & $\begin{array}{c}\text { Flavonols, flavanols, anthocyanins, } \\
\text { isoflavones, phenolic acid }\end{array}$ \\
Trace elements & $\longrightarrow$ & Selenium, copper, manganese, zinc \\
\hline
\end{tabular}

agent and subsequently decrease its efficacy [21, 49]. On the other hand, others argue that antioxidant supplements are beneficial to patients undergoing chemotherapy because they enhance the efficacy of the chemotherapy as well as alleviate toxic side effects, allowing patients to tolerate chemotherapy for the full course of treatment and lessen the need for dose reduction [20,43]. Despite convincing 
evidence from preclinical experiments, clinical trials that tested dietary antioxidant nutrients and micronutrients as cancer chemoprevention agents have been unsuccessful or even resulted in harm $[49,50]$. The lack of success in clinical trials and the discrepancies with preclinical experiments can be explained by factors, such as (i) lack of biological rationale for selecting the specific agents of interest, (ii) limited number of agents tested to date, and (iii) insufficient duration of the interventions and follow-up. Moreover, this high level of heterogeneity within epidemiological data may support the existence of other factors that could modulate the relationship between antioxidant and cancer development, explaining contrasted results across different populations [21, 49].

\subsection{Endogenous Antioxidants. Modulation of endogenous} antioxidants is among other strategies to balance the intracellular redox levels. Among the various ROS metabolically generated, $\mathrm{H}_{2} \mathrm{O}_{2}$, the nonradical two-electron reduction product of oxygen, emerged as central hub in redox signaling and oxidative stress. Processes such as proliferation, differentiation, inflammation, and apoptosis use $\mathrm{H}_{2} \mathrm{O}_{2}$ as signaling compound. Metabolic sinks of this low-molecular-weight include the peroxidatic reaction carried out by catalase and numerous peroxidases [51]. However, due to the high affinity of $\mathrm{H}_{2} \mathrm{O}_{2}$ for thiol residues, the new and expanding family of thiol-specific antioxidant enzymes, peroxiredoxins, has received considerable attention. Indeed, under physiological conditions, eukaryotic peroxiredoxins are responsible for the reduction of $90 \%$ of intracellular $\mathrm{H}_{2} \mathrm{O}_{2}$. On the other hand, peroxiredoxins can be easily inactivated by $\mathrm{H}_{2} \mathrm{O}_{2}$, disabling peroxidase activity and therefore limiting their ability to act as antioxidant, particularly in an oxidative environment like inflammation and intermittent hypoxia [52]. Notably, enhancement of GSH levels was described in hypoxic intracellular environment $[53,54]$. Glutathione (GSH) is considered to be the major thiol-disulfide redox buffer of the cell. On average, the GSH intracellular concentration is $0.5-10 \mathrm{mM}$ [55]. This is far higher than most redox active compounds making GSH an important intracellular antioxidant and redox potential regulator that plays a vital role in drug detoxification and cellular protection from damage by free radicals, peroxides, and toxins [56]. Given the range of critical cellular functions involving GSH, it has long been considered that the modulation of intracellular GSH levels would be of great clinical benefits. Enhancement of GSH levels for cytoprotection is available by the administration of its precursor $\mathrm{N}$-Acetyl cysteine, since direct administration of reduced GSH has physical and chemical limitations [56, 57]. Contrastingly, these cytoprotective effects of GSH and its associated enzymes in many types of cancer lead to an increased tumor cell survival and chemotherapy drug resistance [58].

4.2.1. Glutathione and Chemoresistance. Preclinical studies of chemosensitization through antioxidant modulation have been reported in different tumor cells [44, 58-60]. However, chemoresistance is a complex system with multiple and heterogeneous mechanisms of action which are orchestrated not only by the tumor microenvironments but also by the biology of the tumor $[61,62]$. Although most of the chemotherapeutic drugs are prooxidants, not all the cancer cell death induction pathways are ROS-dependent [43]. Nevertheless, chemoresistance is not caused by a single factor but rather contributed by combinations of many drug-resistant factors such as (1) reduced intracellular drug accumulation which may result from changes in drug transportation (increased efflux and decreased influx of anticancer drugs) and/or enhancement of detoxification activity; (2) increased DNA repair involving increased nucleotide excision repair, interstrand crosslink repair, or loss mismatch repair; (3) changes in the apoptotic cell death pathways; and (4) intracellular elevated antioxidant levels [63]. Among the antioxidants involved in the maintenance of intracellular redox balance, a main role is played by glutathione (GSH) [57]. GSH and its related enzymes participate not only in the antioxidant defense systems, but also in some drug-resistance metabolic processes such as detoxification and efflux of xenobiotics and blockage of the apoptosis tumor cell death pathway [58]. GSH is a major contributing factor to drug resistance by interacting with chemotherapeutic drugs such as cisplatin and trisenox [64, 65]. In fact, there are clinical evidences supporting a role of the GSH system in overcoming drug resistance and/or toxicity in solid tumors (e.g., lung cancers and bladder) treatments outcome $[60,63]$. Therefore, much effort has been directed at depleting cellular GSH levels in order to sensitize tumor cells to the cytotoxic effects of anticancer drugs. The use of buthionine sulfoximine (BSO), an inhibitor of GSH synthesis [66], was performed in clinical trials [67-70]. However, the approach was limited by BSO availability and lack of selectivity of this drug for tumor versus normal cells [56]. But it is notable that GSH plays an important role in drug resistance and its depletion demonstrated to be effective in the sensitization of different types of cancer patients to cytotoxic chemotherapy [67-70]. Another alternative in progress is the development and optimization of GSH analogues that inhibit the enzyme glutathione-S-transferase (GST) responsible for the detoxification overcoming, therefore, chemoresistance $[56,71]$. Among the GSH analogues developed, one (TLK 286), which is in clinical trial phase 3 settings for non-smallcell lung and ovarian cancer, appears to sensitize these tumors to cytotoxic chemotherapies [56]. However, the lack of tumor specificity is still a potential problem.

4.3. Antioxidant and Possible Clinical Benefits. Altogether, it should be recognized that understanding the redox biochemistry differences between normal and cancer cells is essential for the design and development of strategies to overcome oxidative damage or prooxidant chemoresistance $[61,62]$. Even within a specific cancer type, the malignant cell populations are heterogeneous and intracellular oxidative levels may change as the disease progress [61]. Consequently, studying intra- and intertumor heterogeneous distribution of antioxidants levels may be an important factor to overcome tumor progression. Additionally, it is known that alterations in cellular redox metabolism play a crucial role in the activation or loss of tumor suppressor proteins activities such as breast cancer susceptibility gene breast cancer 1 (BRCA1) and phosphatase and tensin homolog deleted on chromosome 10 




FIgURE 5: Possible clinical benefits of antioxidant in tumor progression.

(PTEN) [3, 72-78]. The BRCA1 is an oncosuppressor gene with a relatively broad cellular role such as DNA repair (including nucleotide excision repair, NER, and doublestrand break repair, DSBR), transcriptional regulation, and chromatin remodeling [79-83]. Notably, BRCA1 has also an antioxidant role in response to oxidative stress in which ROS cause DNA damage due to oxidation [73-75]. Loss or mutation of the BRCA1 gene was firstly described to be associated with increased risk of breast and ovarian cancers [80, 82-86]. Moreover, BRCA1 expression has been correlated with cancer aggressiveness and chemotherapy sensitivity in other solid tumors such as prostate [87-89], non-small-cell lung cancer [90-94], and pancreas [95, 96]. The superfamily of protein tyrosine phosphatase (PTP) enzymes functions in a coordinated pattern with protein tyrosine kinases to control the cellular regulatory signal processes such as cell growth, proliferation, and differentiation [97-100]. PTEN, a class 2 VH1-like (poxvirus vaccinia) DUSP (dual specificity phosphatase) [100] and likewise a member of phosphatase protein family, is modulated by ROS $[98,101]$. The oxidation of the active site Cys by ROS abrogates PTEN catalytic activity and, thereby, switching on the phosphatidylinositol-3-kinase (PI3K) proliferation pathway $[72,75-77,100,101]$. The tumor suppressor PTEN is one of the most frequently mutated genes in human cancer and is generally associated with advanced cancers and metastases [72]. A recent study reveals that PTEN loss activity is the most important alteration for cellular malignant transformation in mammary epithelial cells [102]. A recent study reveals that PTEN loss activity is a common event in breast cancers caused by BRCA1 deficiency [103] due to ROS enhancement. Recently, the modulation of other tumor suppressor genes was described to be ROS-dependent $[78,104,105]$. Therefore, the use of antioxidant might protect the biomarkers ROS-dependent tumorigenesis like BRCA1 and PTEN (Figure 5).

\section{Conclusions}

Enhancing the capacity of antioxidant in order to protect cells from redox-related changes or environmental toxins represents a persistent aim in the search for cytoprotective strategies against cancer. On the contrary, the strategy of depleting antioxidant is aimed at sensitizing cancer cells to chemotherapy, the so-called chemosensitization. In this context, it has been reported that antioxidant may be a determining factor for the sensitivity of some tumors to various chemotherapeutic agents. In particular, GSH and GSH enzyme-linked system are a relevant parameter for chemotherapy response, and it may be utilized as a useful biomarker for selecting tumors potentially responsive to chemotherapeutic regiments. The involvement of antioxidant in the carcinogenesis and in the drug resistance of tumor cell is clear, but further studies, aimed at understanding the antioxidant-driven molecular pathways and the biology of the tumor cells, are crucial to design new therapeutic strategies to fight cancer progression and overcome chemoresistance.

\section{Conflict of Interests}

The author declares that there is no conflict of interests regarding the publication of this paper.

\section{Acknowledgments}

The author would like to express the deepest gratitude to A. C. S. Ferreira, B. M. Bomfim, L. F. R. Silva, and R. A. Fernandes, who believed in their project from the start and, in particular, to D. F. Baptista to whom this paper is dedicated. This work was supported by grants from Fundação do Câncer (FAF).

\section{References}

[1] J. P. Fruehauf and F. L. Meyskens Jr., "Reactive oxygen species: a breath of life or death?" Clinical Cancer Research, vol. 13, no. 3, pp. 789-794, 2007.

[2] M. L. Circu and T. Y. Aw, "Reactive oxygen species, cellular redox systems, and apoptosis," Free Radical Biology and Medicine, vol. 48, no. 6, pp. 749-762, 2010.

[3] T. Finkel, "Signal transduction by reactive oxygen species," Journal of Cell Biology, vol. 194, no. 1, pp. 7-15, 2011.

[4] M. Dodson, V. Darley-Usmar, and J. Zhang, "Cellular metabolic and autophagic pathways: traffic control by redox signaling," Free Radical Biology and Medicine, vol. 63, pp. 207-221, 2013. 
[5] M. Jimenez-Del-Rio and C. Velez-Pardo, "The bad, the good, and the ugly about oxidative stress," Oxidative Medicine and Cellular Longevity, vol. 2012, Article ID 163913, 13 pages, 2012.

[6] V. A. Kobliakov, "Mechanisms of tumor promotion by reactive oxygen species," Biochemistry (Moscow), vol. 75, no. 6, pp. 675$685,2010$.

[7] J. S. Beckman and W. H. Koppenol, "Nitric oxide, superoxide, and peroxynitrite: the good, the bad, and the ugly," American Journal of Physiology: Cell Physiology, vol. 271, no. 5, pp. C1424C1437, 1996.

[8] J. S. Stamler, D. J. Singel, and J. Loscalzo, "Biochemistry of nitric oxide and its redox-activated forms," Science, vol. 258, no. 5090, pp. 1898-1902, 1992.

[9] J. Loscalzo, "The identification of nitric oxide as endotheliumderived relaxing factor," Circulation Research, vol. 113, no. 2, pp. 100-103, 2013.

[10] I. Fridovich, "Oxygen toxicity: a radical explanation," The Journal of Experimental Biology, vol. 201, no. 8, pp. 1203-1209, 1998.

[11] B. Kalyanaraman, "Teaching the basics of redox biology to medical and graduate students: oxidants, antioxidants and disease mechanisms," Redox Biology, vol. 1, no. 1, pp. 244-257, 2013.

[12] L. F. Olsen, O. G. Issinger, and B. Guerra, "The yin and yang of redox regulation," Redox Report, vol. 18, no. 6, pp. 245-252, 2013.

[13] P. Ježek and L. Hlavatá, "Mitochondria in homeostasis of reactive oxygen species in cell, tissues, and organism," The International Journal of Biochemistry and Cell Biology, vol. 37, no. 12, pp. 2478-2503, 2005.

[14] R. O. Poyton, K. A. Ball, and P. R. Castello, "Mitochondrial generation of free radicals and hypoxic signaling," Trends in Endocrinology and Metabolism, vol. 20, no. 7, pp. 332-340, 2009.

[15] C. L. Quinlan, I. V. Perevoshchikova, M. Hey-Mogensen et al., "Sites of reactive oxygen species generation by mitochondria oxidizing different substrates," Redox Biology, vol. 1, no. 1, pp. 304-312, 2013.

[16] J. D. Lambeth, T. Kawahara, and B. Diebold, "Regulation of Nox and Duox enzymatic activity and expression," Free Radical Biology and Medicine, vol. 43, no. 3, pp. 319-331, 2007.

[17] R. P. Brandes, N. Weissmann, and K. Schröder, "Nox family NADPH oxidases in mechano-transduction: mechanisms and consequences," Antioxidants and Redox Signaling, vol. 20, no. 6, pp. 887-898, 2014.

[18] N. Cantu-Medellin and E. E. Kelley, "Xanthine oxidoreductasecatalyzed reactive species generation: a process in critical need of reevaluation," Redox Biology, vol. 1, no. 1, pp. 353-358, 2013.

[19] J. Lugrin, N. Rosenblatt-Velin, R. Parapanov et al., "The role of oxidative stress during inflammatory processes," Biological Chemistry, vol. 395, no. 2, pp. 203-230, 2014.

[20] V. Fuchs-Tarlovsky, "Role of antioxidants in cancer therapy," Nutrition, vol. 29, no. 1, pp. 15-29, 2012.

[21] K. I. Block, A. C. Koch, M. N. Mead, P. K. Tothy, R. A. Newman, and C. Gyllenhaal, "Impact of antioxidant supplementation on chemotherapeutic toxicity: a systematic review of the evidence from randomized controlled trials," International Journal of Cancer, vol. 123, no. 6, pp. 1227-1239, 2008.

[22] J. Denekamp, A. Daşu, and A. Waites, "Vasculature and microenvironmental gradients: the missing links in novel approaches to cancer therapy?" Advances in Enzyme Regulation, vol. 38, no. 1, pp. 281-299, 1998.

[23] C. Cooper, G.-Y. Liu, Y.-L. Niu, S. Santos, L. C. Murphy, and P. H. Watson, "Intermittent hypoxia induces proteasome-depe- ndent down-regulation of estrogen receptor $\alpha$ in human breast carcinoma," Clinical Cancer Research, vol. 10, no. 24, pp. 87208727, 2004.

[24] C.-H. Hsieh, C.-H. Lee, J.-A. Liang, C.-Y. Yu, and W.-C. Shyu, "Cycling hypoxia increases U87 glioma cell radioresistance via ROS induced higher and long-term HIF-1 signal transduction activity," Oncology Reports, vol. 24, no. 6, pp. 1629-1636, 2010.

[25] Y. Liu, X. Song, X. Wang et al., "Effect of chronic intermittent hypoxia on biological behavior and hypoxia-associated gene expression in lung cancer cells," Journal of Cellular Biochemistry, vol. 111, no. 3, pp. 554-563, 2010.

[26] V. K. Bhaskara, I. Mohanam, J. S. Rao, and S. Mohanam, "Intermittent hypoxia regulates stem-like characteristics and differentiation of neuroblastoma cells," PLoS ONE, vol. 7, no. 2, Article ID e30905, 2012.

[27] K. A. Whelan and M. J. Reginato, "Surviving without oxygen: hypoxia regulation of mammary morphogenesis and anoikis," Cell Cycle, vol. 10, no. 14, pp. 2287-2294, 2011.

[28] S. E. Rademakers, P. N. Span, J. H. A. M. Kaanders, F. C. G. J. Sweep, A. J. van der Kogel, and J. Bussink, "Molecular aspects of tumour hypoxia," Molecular Oncology, vol. 2, no. 1, pp. 41-53, 2008.

[29] S. Strese, M. Fryknäs, R. Larsson et al., "Effects of hypoxia on human cancer cell line chemosensitivity," BMC Cancer, vol. 13, no. 331, pp. 1-11, 2013.

[30] Y. Yang, S. Karakhanova, J. Werner et al., "Reactive oxygen species in cancer biology and anticancer therapy," Current Medicinal Chemistry, vol. 20, no. 30, pp. 3677-3692, 2013.

[31] C. H. Hsieh, C. P. Wu, H. T. Lee et al., "NADPH oxidase subunit 4 mediates cycling hypoxia-promoted radiation resistance in glioblastoma multiforme," Free Radical Biology and Medicine, vol. 53, no. 4, pp. 649-658, 2012.

[32] L. Flamant, E. Roegiers, M. Pierre et al., “TMEM45A is essential for hypoxia-induced chemoresistance in breast and liver cancer cells," BMC Cancer, vol. 12, no. 391, pp. 1-16, 2012.

[33] T. Rhim, D. Y. Lee, and M. Lee, "Hypoxia as target for tissue specific gene therapy," Journal of Controlled Release, vol. 172, no. 2, pp. 484-494, 2013.

[34] T. Miyata, S. Takizawa, and C. van Ypersele de Strihou, "Hypoxia. 1. Intracellular sensors for oxygen and oxidative stress: novel therapeutic targets," American Journal of Physiology: Cell Physiology, vol. 300, no. 2, pp. C226-C231, 2011.

[35] J.-W. Kim, P. Gao, and C. V. Dang, "Effects of hypoxia on tumor metabolism," Cancer and Metastasis Reviews, vol. 26, no. 2, pp. 291-298, 2007.

[36] J. Nanduri, N. Wang, G. Yuan et al., "Intermittent hypoxia degrades HIF- $2 \alpha$ via calpains resulting in oxidative stress: implications for recurrent apnea-induced morbidities," Proceedings of the National Academy of Sciences of the United States of America, vol. 106, no. 4, pp. 1199-1204, 2009.

[37] B. Keith, R. S. Johnson, and M. C. Simon, "HIF1 $\alpha$ and HIF2 $\alpha$ : sibling rivalry in hypoxic tumour growth and progression," Nature Reviews Cancer, vol. 12, no. 1, pp. 9-22, 2012.

[38] J. Nanduri, D. R. Vaddi, S. A. Khan et al., "Xanthine oxidase mediates hypoxia-inducible factor- $2 \alpha$ degradation by intermittent hypoxia," PLoS ONE, vol. 8, no. 10, Article ID e75838, 2013.

[39] H. Rundqvist and R. S. Johnson, "Tumour oxygenation: implications for breast cancer prognosis," Journal of Internal Medicine, vol. 274, no. 2, pp. 105-112, 2013.

[40] B. Ramanathan, K.-Y. Jan, C.-H. Chen, T.-C. Hour, H.-J. Yu, and Y.-S. Pu, "Resistance to paclitaxel is proportional to cellular total 
antioxidant capacity," Cancer Research, vol. 65, no. 18, pp. 84558460, 2005.

[41] C. M. Woolston, L. Zhang, S. J. Storr et al., "The prognostic and predictive power of redox protein expression for anthracyclinebased chemotherapy response in locally advanced breast cancer," Modern Pathology, vol. 25, no. 8, pp. 1106-1116, 2012.

[42] A. Brozovic, A. Ambriović-Ristov, and M. Osmak, "The relationship between cisplatin-Induced reactive oxygen species, glutathione, and BCL-2 and resistance to cisplatin," Critical Reviews in Toxicology, vol. 40, no. 4, pp. 347-359, 2010.

[43] D. T. Vincent, Y. F. Ibrahim, M. G. Espey et al., "The role of antioxidant in the era of cardio-oncology," Cancer Chemotherapy and Pharmacology, vol. 72, no. 6, pp. 1157-1168, 2013.

[44] C. Glorieux, N. Dejeans, B. Sid, R. Beck, P. B. Calderon, and J. Verrax, "Catalase overexpression in mammary cancer cells leads to a less aggressive phenotype and an altered response to chemotherapy," Biochemical Pharmacology, vol. 82, no. 10, pp. 13841390, 2011.

[45] E. M. Hanschmann, J. R. Godoy, C. Berndt et al., "Thioredoxins, glutaredoxins, and peroxiredoxins-molecular mechanisms and health significance: from cofactors to antioxidants to redox signaling," Antioxidants and Redox Signaling, vol. 19, no. 13, pp. 1539-1605, 2013.

[46] D. D. Rio, A. R. Mateos, J. P. E. Spencer et al., "Dietary (poly) phenolics in human health: structures, bioavailability, and evidence of protective effects against chronic diseases," Antioxidants and Redox Signaling, vol. 18, no. 14, pp. 1818-1892, 2013.

[47] M. Zalewska, J. Trefon, and H. Milnorowicz, "The role of metallothionein interactions with other proteins," Proteomics, 2014.

[48] I. Romero-Canelón and P. J. Sadler, "Next-generation metal anticancer complexes: multitargeting via redox modulation," Inorganic Chemistry, vol. 52, no. 21, pp. 12276-12291, 2013.

[49] M. Goodman, R. M. Bostick, O. Kucuk, and D. P. Jones, "Clinical trials of antioxidants as cancer prevention agents: past, present, and future," Free Radical Biology and Medicine, vol. 51, no. 5, pp. 1068-1084, 2011.

[50] G. Bjelakovic, D. Nikolova, and C. Gluud, "Antioxidant supplements and mortality," Current Opinion in Clinical Nutrition and Metabolic Care, vol. 17, no. 1, pp. 40-44, 2014.

[51] H. Sies, "Role of metabolic $\mathrm{H}_{2} \mathrm{O}_{2}$ generation: redox signaling and oxidative stress," The Journal Biological Chemistry, 2014.

[52] R. A. Poynton and M. B. Hampton, "Peroxiredoxins as biomarkers of oxidative stress.," Biochimica et Biophysica Acta, vol. 1840, no. 2, pp. 906-912, 2014.

[53] J. J. Merino, C. Roncero, M. J. Oset-Gasque et al., "Antioxidant and protective mechanisms against hypoxia and hypoglycaemia in cortical neurons in vitro," International Journal of Molecular Sciences, vol. 15, no. 2, pp. 2475-2493, 2014.

[54] V. V. Khramtsov and R. J. Gillies, "Janus-faced tumor microenvironment and redox," Antioxidant and Redox Signaling, 2014.

[55] V. I. Lushchak, "Glutathione homeostasis and fuctions: potential targets for medical interventions," Journal of Amino Acids, vol. 2012, Article ID 736837, 26 pages, 2012.

[56] J. H. Wu and G. Batist, "Glutathione and glutathione analogues, therapeutic potentials," Biochimica et Biophysica Acta, vol. 1830, no. 5, pp. 3350-3353, 2013.

[57] D. M. Townsend, K. D. Tew, and H. Tapiero, "The importance of glutathione in human disease," Biomedicine and Pharmacotherapy, vol. 57, no. 3, pp. 145-155, 2003.

[58] N. Traverso, R. Ricciarelli, M. Nitti et al., "Role of glutathione in cancer progression and chemoresistance," Oxidative Medicine and Cellular Longevity, vol. 2013, Article ID 972913, 10 pages, 2013.

[59] J. K. Kwee, D. G. Luque, A. C. D. S. Ferreira et al., "Modulation of reactive oxygen species by antioxidants in chronic myeloid leukemia cells enhances imatinib sensitivity through survivin downregulation," Anti-Cancer Drugs, vol. 19, no. 10, pp. 975-981, 2008.

[60] P. Yang, J. O. Ebbert, Z. Sun, and R. M. Weinshilboum, "Role of the glutathione metabolic pathway in lung cancer treatment and prognosis: a review," Journal of Clinical Oncology, vol. 24, no. 11, pp. 1761-1769, 2006.

[61] D. Hanahan and R. A. Weinberg, "Hallmarks of cancer: the next generation,” Cell, vol. 144, no. 5, pp. 646-674, 2011.

[62] T. Fiaschi and P. Chiarugi, "Oxidative stress, tumor microenvironment, and metabolic reprogramming: a diabolic liaison," International Journal of Cell Biology, vol. 2012, Article ID 762825, 8 pages, 2012.

[63] X. Hu and Y. Xuan, "Bypassing cancer drug resistance by activating multiple death pathways - a proposal from the study of circumventing cancer drug resistance by induction of necroptosis," Cancer Letters, vol. 259, no. 2, pp. 127-137, 2008.

[64] B. Köberle, M. T. Tomicic, S. Usanova, and B. Kaina, "Cisplatin resistance: preclinical findings and clinical implications," Biochimica et Biophysica Acta, vol. 1806, no. 2, pp. 172-182, 2010.

[65] P.-S. Ong, S.-Y. Chan, and P. C. Ho, "Differential augmentative effects of buthionine sulfoximine and ascorbic acid in $\mathrm{As}_{2} \mathrm{O}_{3}$-induced ovarian cancer cell death: Oxidative stressindependent and -dependent cytotoxic potentiation," International Journal of Oncology, vol. 38, no. 6, pp. 1731-1739, 2011.

[66] M. Jozefczak, T. Remans, J. Vangronsveld, and A. Cuypers, "Glutathione is a key player in metal-induced oxidative stress defenses," International Journal of Molecular Sciences, vol. 13, no. 3, pp. 3145-3175, 2012.

[67] H. H. Bailey, R. T. Mulcahy, K. D. Tutsch et al., "Phase I clinical trial of intravenous L-buthionine sulfoximine and melphalan: an attempt at modulation of glutathione," Journal of Clinical Oncology, vol. 12, no. 1, pp. 194-205, 1994.

[68] P. J. O’Dwyer, T. C. Hamilton, F. P. LaCreta et al., "Phase I trial of buthionine sulfoximine in combination with melphalan in patients with cancer," Journal of Clinical Oncology, vol. 14, no. 1, pp. 249-256, 1996.

[69] H. H. Bailey, G. Ripple, K. D. Tutsch et al., "Phase I study of continuous-infusion L-S,R-buthionine sulfoximine with intravenous melphalan," Journal of the National Cancer Institute, vol. 89, no. 23, pp. 1789-1796, 1997.

[70] H. H. Bailey, "L-S,R-buthionine sulfoximine: historical development and clinical issues," Chemico-Biological Interactions, vol. 111-112, pp. 239-254, 1998.

[71] D. Hamilton and G. Batist, "Glutathione analogues in cancer treatment," Current Oncology Reports, vol. 6, no. 2, pp. 116-122, 2004.

[72] L. Salmena, A. Carracedo, and P. P. Pandolfi, “Tenets of PTEN tumor suppression," Cell, vol. 133, no. 3, pp. 403-414, 2008.

[73] T. Saha, J. K. Rih, and E. M. Rosen, "BRCA1 down-regulates cellular levels of reactive oxygen species," The FEBS Letters, vol. 583, no. 9, pp. 1535-1543, 2009.

[74] Z. Huang, L. Zuo, Z. Zhang et al., “3,3'-Diindolylmethane decreases VCAM-1 expression and alleviates experimental colitis via a BRCA1-dependent antioxidant pathway," Free Radical Biology and Medicine, vol. 50, no. 2, pp. 228-236, 2011. 
[75] B. Vurusaner, G. Poli, and H. Basaga, "Tumor suppressor genes and ROS: complex networks of interactions," Free Radical Biology and Medicine, vol. 52, no. 1, pp. 7-18, 2012.

[76] H. Luo, Y. Yang, J. Duan et al., "PTEN-regulated AKT/FoxO3a/ Bim signaling contributes to reactive oxygen species-mediated apoptosis in selenite-treated colorectal cancer cells," Cell Death and Disease, vol. 4, article e481, 2013.

[77] F. G. Meng and Z. Y. Zhang, "Redox regulation of protein tyrosine phosphatase activity by hydroxyl radical," Biochimica et Biophysica Acta, vol. 1834, no. 1, pp. 464-469, 2013.

[78] S. J. Kim, H. J. Jung, and C. J. Lim, "Reactive oxygen speciesdependent down-regulation of tumor suppressor genes PTEN, USP28, DRAM, TIGAR, and CYLD under oxidative stress," Biochemical Genetics, vol. 51, no. 11-12, pp. 901-915, 2013.

[79] A. Tutt and A. Ashworth, "The relationship between the roles of BRCA genes in DNA repair and cancer predisposition," Trends in Molecular Medicine, vol. 8, no. 12, pp. 571-576, 2002.

[80] N. Turner, A. Tutt, and A. Ashworth, "Hallmarks of'BRCAness' in sporadic cancers," Nature Reviews Cancer, vol. 4, no. 10, pp. 814-819, 2004.

[81] R. Roy, J. Chun, and S. N. Powell, "BRCA1 and BRCA2: different roles in a common pathway of genome protection," Nature Reviews Cancer, vol. 12, no. 1, pp. 68-78, 2012.

[82] M. L. Li and R. A. Greenberg, "Links between genome integrity and BRCA1 tumor suppression," Trends in Biochemical Sciences, vol. 37, no. 10, pp. 418-424, 2012.

[83] W. D. Foulkes and A. Y. Shuen, "In brief: BRCA1 and BRCA2," Journal of Pathology, vol. 230, no. 4, pp. 347-349, 2013.

[84] Y. Miki, J. Swensen, D. Shattuck-Eidens et al., "A strong candidate for the breast and ovarian cancer susceptibility gene BRCA1," Science, vol. 266, no. 5182, pp. 66-71, 1994.

[85] P. A. Futreal, Q. Liu, D. Shattuck-Eidens et al., "BRCA1 mutations in primary breast and ovarian carcinomas," Science, vol. 266, no. 5182, pp. 120-122, 1994.

[86] N. J. Birkbak, B. Kochupurakkai, J. M. G. Izarzugaza et al., "Tumor mutation burden forecasts outcome in ovarian cancer with BRCA1 or BRCA2 mutations," PLos ONE, vol. 08, no. 11, Article ID e80023, 2013.

[87] S. Fan, J.-A. Wang, R.-Q. Yuan et al., "BRCA1 as a potential human prostate tumor suppressor: modulation of proliferation, damage responses and expression of cell regulatory proteins," Oncogene, vol. 16, no. 23, pp. 3069-3082, 1998.

[88] E. Castro, C. Goh, D. Olmos et al., "Germline BRCA mutations are associated with higher risk of nodal involvement, distant metastasis, and poor survival outcomes in prostate cancer," Journal of Clinical Oncology, vol. 31, no. 14, pp. 1748-1757, 2013.

[89] P. de Luca, C. P. Moiola, F. Zalazar et al., "BRCA1 and p53 regulate critical prostate cancer pathways," Prostate Cancer and Prostatic Diseases, vol. 16, no. 3, pp. 233-238, 2013.

[90] A. Passaro, A. Palazzo, P. Trenta et al., "Molecular and clinical analysis of predictive biomarkers in non-small-cell lung cancer," Current Medicinal Chemistry, vol. 19, no. 22, pp. 3689-3700, 2012.

[91] M. Pesta, V. Kulda, O. Fiala et al., "Prognostic significance of ERCC1, RRM1 and BRCA1 in surgically-treated patients with non-small cell lung cancer," Anticancer Research, vol. 32, no. 11, pp. 5003-5010, 2012.

[92] H. Harada, K. Miyamoto, Y. Yamashita et al., "Methylation of breast cancer susceptibility gene 1 (BRCA1) predicts recurrence in patients with curatively resected stage I non-small cell lung cancer," Cancer, vol. 119, no. 4, pp. 792-798, 2013.
[93] M. Tiseo, P. Bordi, B. Bortesi et al., "ERCC1/BRCA1 expression and gene polymorphisms as prognostic and predictive factors in advanced NSCLC treated with or without cisplatin," British Journal of Cancer, vol. 108, no. 8, pp. 1695-1703, 2013.

[94] M. Sung and P. Giannakakou, "BRCA1 regulates microtubule dynamics and taxane-induced apoptotic cell signaling," Oncogene, vol. 33, no. 11, pp. 1418-1428, 2014.

[95] J. E. Axilbund and E. A. Wiley, "Genetic testing by cancer site: pancreas," Cancer Journal, vol. 18, no. 4, pp. 350-354, 2012.

[96] T. Wang, S. C. Wentz, N. L. Ausborn et al., "Pattern of breast cancer susceptibility gene 1 expression is a potential prognostic biomarker in resectable pancreatic ductal adenocarcinoma," Pancreas, vol. 42, no. 6, pp. 977-982, 2013.

[97] E. B. Fauman and M. A. Saper, "Structure and function of the protein tyrosine phosphatases," Trends in Biochemical Sciences, vol. 21, no. 11, pp. 413-417, 1996.

[98] N. K. Tonks, "Protein tyrosine phosphatases: from genes, to function, to disease," Nature Reviews Molecular Cell Biology, vol. 7, no. 11, pp. 833-846, 2006.

[99] T. Hunter, "Tyrosine phosphorylation: thirty years and counting," Current Opinion in Cell Biology, vol. 21, no. 2, pp. 140-146, 2009.

[100] L. Tautz, D. A. Critton, and S. Grotegut, "Protein tyrosine phosphatases: structure, function, and implication in human disease," Methods in Molecular Biology, vol. 1053, pp. 179-221, 2013.

[101] N. K. Tonks, "Redox redux: revisiting PTPs and the control of cell signaling," Cell, vol. 121, no. 5, pp. 667-670, 2005.

[102] M. M. Pires, B. D. Hopkins, L. H. Saal et al., "Alterations of EGFR, p53 and PTEN that mimic changes found in basallike breast cancer promote transformation of human mammary epithelial cells," Cancer Biology and Therapy, vol. 14, no. 3, pp. 246-253, 2013.

[103] L. H. Saal, S. K. Gruvberger-Saal, C. Persson et al., "Recurrent gross mutations of the PTEN tumor suppressor gene in breast cancers with deficient DSB repair," Nature Genetics, vol. 40, no. 1, pp. 102-107, 2008.

[104] K. Bensaad, A. Tsuruta, M. A. Selak et al., "TIGAR, a p53-inducible regulator of glycolysis and apoptosis," Cell, vol. 126, no. 1, pp. 107-120, 2006.

[105] K. Bensaad, E. C. Cheung, and K. H. Vousden, "Modulation of intracellular ROS levels by TIGAR controls autophagy," The EMBO Journal, vol. 28, no. 19, pp. 3015-3026, 2009. 

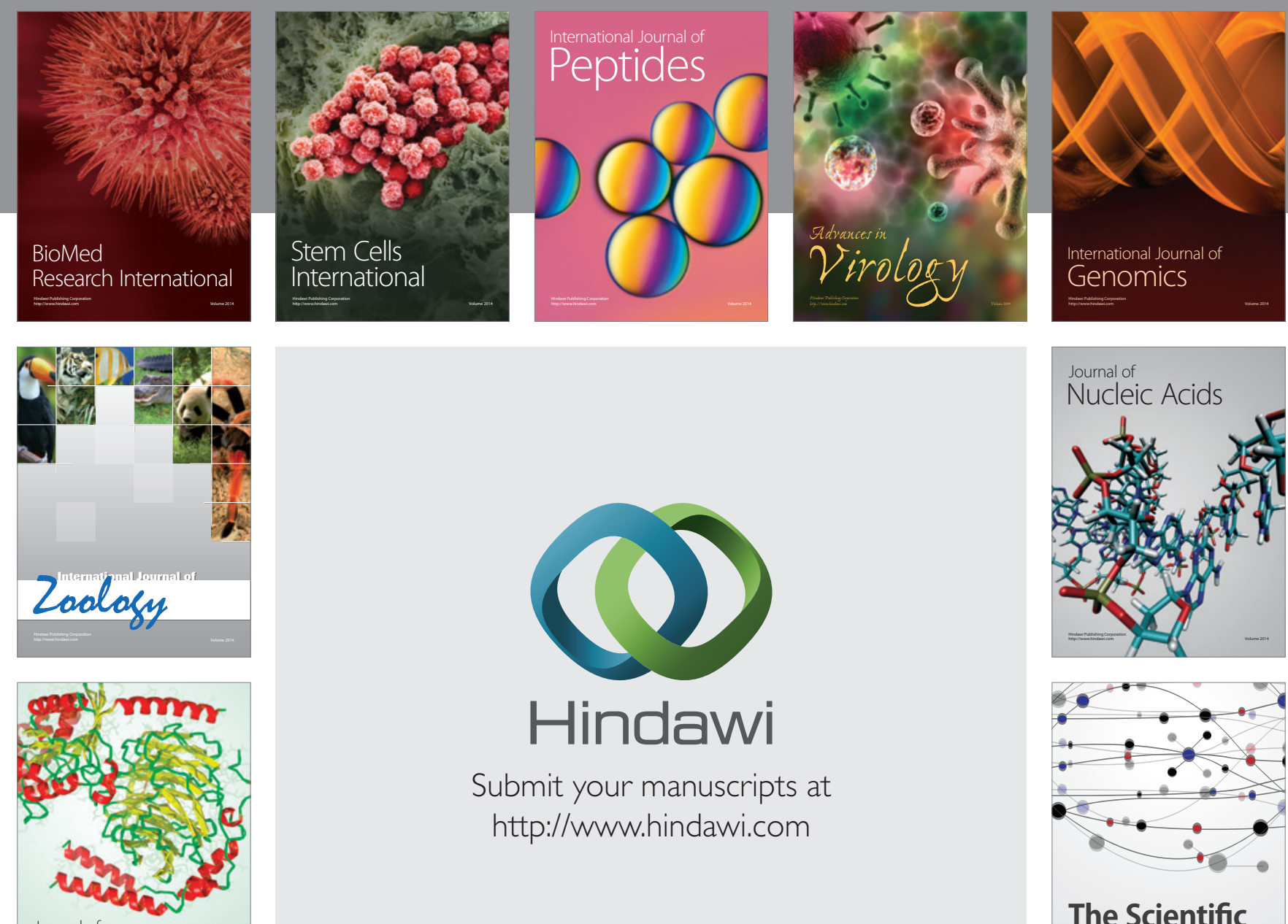

Submit your manuscripts at

http://www.hindawi.com

Journal of
Signal Transduction
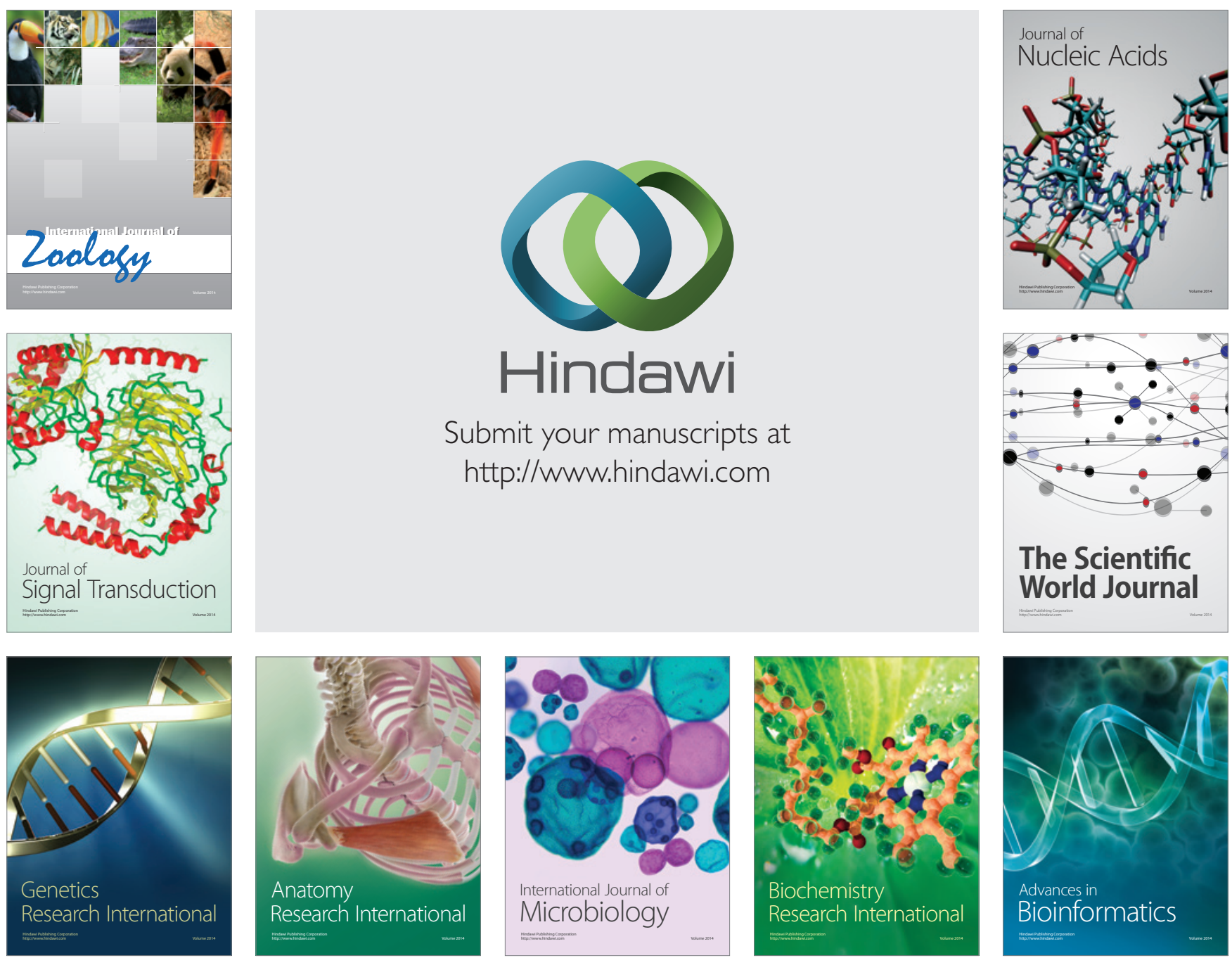

The Scientific World Journal
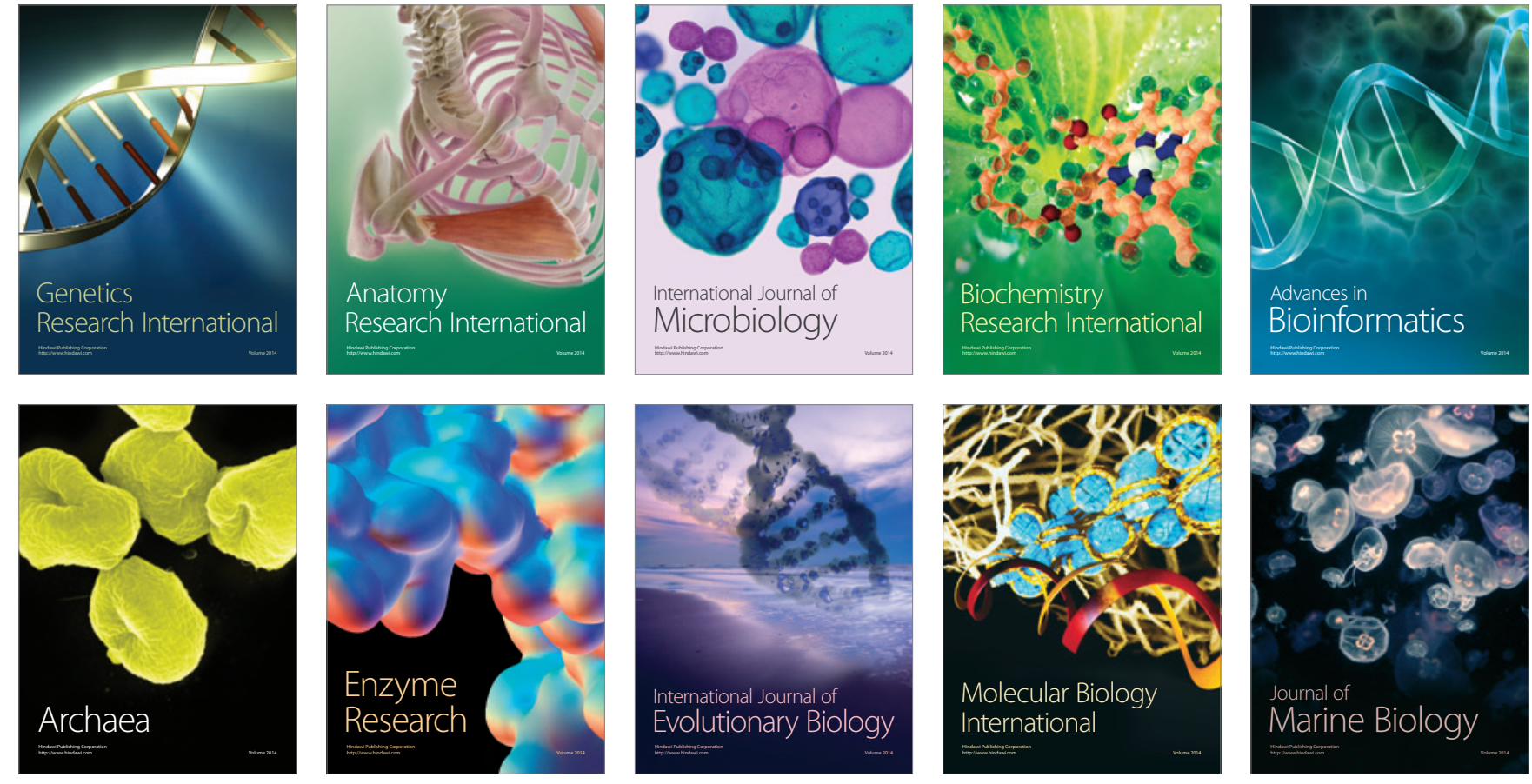